\title{
Development and Implementation of Novel UHF Paper-Based RFID Designs for Anti- counterfeiting and Security Applications
}

\author{
Amin Rida*, Rushi Vyas, Terence Wu, Ronglin Li, and Manos M. Tentzeris \\ PIREAS RFID/Sensors Lab, Georgia Electronic Design Center, School of Electrical and Computer Engineering, \\ Georgia Institute of Technology, Atlanta, GA 30332-0250, USA \\ *E-mail:arida@ece.gatech.edu
}

\section{INTRODUCTION}

Radio Frequency Identification (RFID) is a low-cost compact wireless technology, which is not limited by any Line of Sight $(\mathrm{LoS})$ requirements to communicate with an interrogator and convey information concerning the location, the status and the authentication of an item. RFID allows for simultaneous read/write from/to multiple tags for a variety of propagation environments and shapes. In addition, an easy remote and selective activation or termination of sensor devices based on their unique ID's is possible and allows for the deployment of truly ubiquitous very large ad-hoc networks for ubiquitous sensing, monitoring and security. Due to the increasing demand for automatic identification, the RFID technology has found numerous applications in different areas including inventory tracking and management, retail level management, item level tracking, access control, animal tracking, medical anti-counterfeiting, security, and electronic toll collection.

UHF RFID tags have recently witnessed a tremendous drop in their cost due to the growing interest by the Department of Defense and large retailers such as Wal-Mart. This paper covers those RFID tags operating in the UHF frequency range and whose protocols are set by the Electronic Product Code (EPC) Gen2 protocols worldwide in able to establish and maintain a secure and reliable wireless communication technology. These protocols are preinstalled in RFID interrogators and Integrated Circuits (IC's) of the RFID tags. Compared with the lower frequency tags (LF and HF bands) already suffering from limited read range (1-2 feet), RFID tags in UHF band see the widest use due to their higher read range (over 10 feet) and higher data transfer rate. Among the major challenges that exist in today's RFID technology and potentially delay their large-scale practical implementations are: the design of miniaturized tag antennas with higher efficiency and a better impedance matching between antenna terminals and IC's with typically reactive impedance [1]. This is essential for the maximization of the power flow in passive RFIDs and hence the RFID maximum range and system performance. Another major challenge is the realization of ultra-low-cost RFID tags with individual; cost below 1 cent, something that requires the use of very cheap materials and fabrication processes.

This paper will tackle these challenges by referencing past, present, as well as future work done by the RFID group at Georgia Tech, mainly miniaturizing and matching techniques to overcome the challenge of having a high efficiency match and radiation from a miniaturized antenna, as well as techniques to increase bandwidth and create polarization diversity in UHF antennas, critical attributes for security and anti-counterfeiting applications in global and virtually "environment-independent" level. The second part consists of inkjet printing of RFID modules on organic substrates, such as paper. By this, large reel-to- reel processes can manufacture RFID tags especially on paper which is environmental friendly and one of the cheapest material that is known, enabling RFID use for a variety of conformal shapes ranging from cylindrical medical bottles to rectangular cargo containers and palettes, as well as to circular automotive and aerospace tires.

\section{ANTENNA DESIGN AND APPLICATION}

\section{i. Bandwidth}

The UHF RFID bands range from $860 \mathrm{MHz}$ to $955 \mathrm{MHz}$ [2] on a worldwide basis, due to the fact that different regions such as North America, Europe, or China abide to different regulations such as power emissions, spurious limits of the reader's signal and allowable frequency bands. Half-wavelength antennas are broadly used in RFID applications, because of their omnidirectional nature which is highly desired in "universal/position-independent reception" RFID systems [3]. One of the desired topologies of integrated UHF RFID antennas is demonstrated in Fig. 1.The dimensions of the antenna shown in fig. 1 are: $7.5 \mathrm{~cm} \times 7.5 \mathrm{~m}$ allowing for an integrated battery and/or sensor 
on the top and bottom of the antenna structure, respectively. The matching network consists of a double inductive feed and shorting stub; these stubs modify the antenna's impedance to match any IC impedance value in order to allow for the optimum power flow from IC to antenna or vice versa [3]. It has to be noted that the antenna has to be compact with efficiency close to $100 \%$. This antenna provides a better bandwidth than typical dipole configurations because of the tapering that was performed to its arms. The simulation and the measurement results are shown in fig. 2 , featuring a very good agreement. The bandwidth defined by the VSWR of 2 or alternatively by a Return Loss of $9.6 \mathrm{~dB}$ is ranging from $860 \mathrm{MHZ}$ to $930 \mathrm{MHz}$.

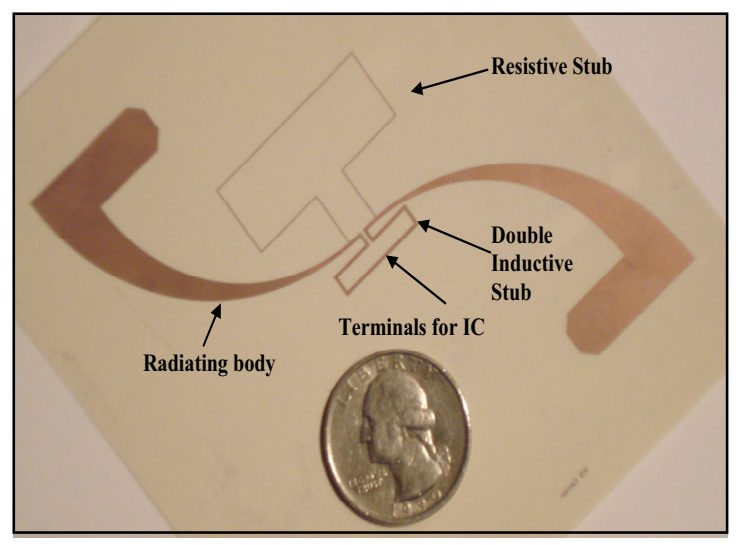

Fig. 1. Typical RFID tag architecture.

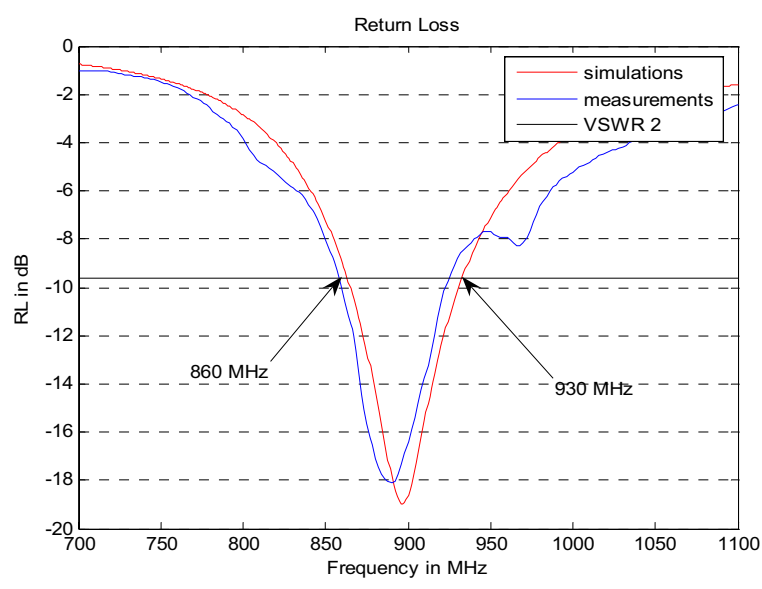

Fig. 2. Return loss of RFID tag shown in fig. 1.

\section{ii. Harsh Environments}

Since most industrial environments, ports, and warehouses are considered as harsh environments due to the presence of metals and/or liquids, RF waves undergo polarization changes. This causes occasional transmission loss or blockage of communication with the RFID reader, which impedes the continuous real-time item tracking or monitoring. The proposed solution is a dual antenna configuration with two identical antennas in dimensions and shape bodies, such as the one shown in Fig. 3. This configuration provides a dual polarization and can significantly minimize polarization changes, while accounting for any misalignment of the RFID tag with respect to the RFID reader that might cause any null in its radiation pattern.

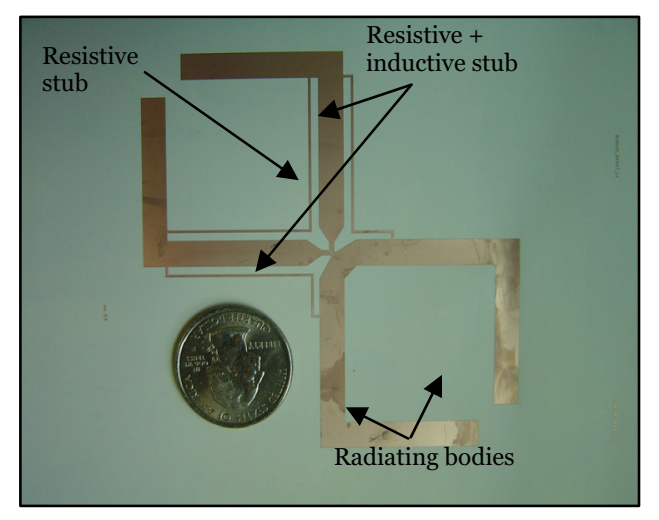

Fig. 3. Dual polarization antenna.

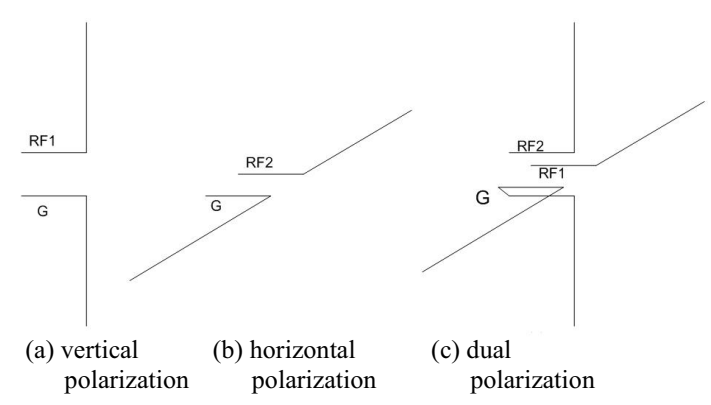

Fig.4. Polarization definition in RFID antennas.

\section{RFID/SENSORS EMBEDDED IN/ON ORGANIC SUBSTRATES: PAPER}

Paper is considered as one of the best organic substrates for RFID applications for several reasons. It is not only environmentally friendly, but it can also undergo large reel-to reel-processing and is one of the cheapest materials 
known. Paper can also host nano-scale additives (i.e. fire retardant textiles) and can be hydrophobic. Most importantly, its dielectric constant $\varepsilon_{\mathrm{r}}$ is close to air's (5-6\% power reflection) allowing for the electromagnetic power to easily penetrate even after the RFID is embedded in the substrate. The low surface profile of paper with an appropriate coating makes paper compatible with fast printing processes, such as conductive paste inkjet printing instead of metal etching techniques, which saves a tremendous amount of time in the fabrication process. In addition, this makes the RFID system much more secure since all fabrication processes can be done 'on site'. This means that the customer may print the design of the RFID module on paper substrates and with an appropriate IC integration process such as surface mount, assembly of a passive RFID tag can be accomplished. This eliminates any chance of counterfeiting ICs and avoiding any modifications (data or memory storage) to be made to ICs before arriving to the customer.

Active tags, requiring the integration of sensors and batteries, can also be printed on paper in a multilayer fashion. Paper substrate can excellently handle high temperature treatment during the assembly process, while its reliability/life time is very high compared to other substrates such as plastic. Paper can also be characterized easily in terms of electrical properties using resonator structures [3]. Dielectric constant and loss tangent can be effectively and accurately measured up to $40 \mathrm{GHz}$ by using methods such as: microstrip ring, cavity and parallel plate resonators [3]. A suggested module of a printed RFID active tag is shown in the figure below. The overall dimensions of the RFID tag shown below are $8.2 \mathrm{~cm} \times 4.5 \mathrm{~cm}$.

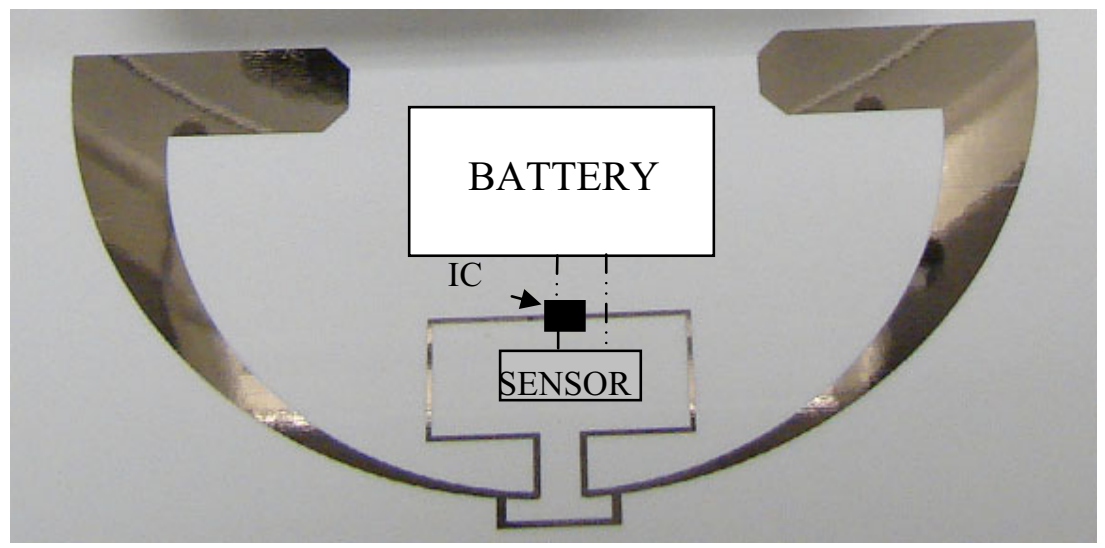

Fig. 5. Suggested outline of an integrated U-shaped antenna with IC, sensor and embedded battery.

\section{RFID APPLICATION IN SECURITY AREAS}

Security is a big concern for profitable and effective business. Theft, for instance, directly results in the losing of revenue. However, facing with a myriad of theft method possibilities and the cost of encountering these, decision makers often despair and have to defer or even ignore the issue of security. RFID technology provides a single solution to deal with the variety of security issues. In this section, three security areas of RFID implementations will be addressed, namely anti-theft, anti-counterfeiting, and national security.

\section{i. Anti-theft}

U.S. retailers lost an estimated $\$ 31.3$ billion from inventory shrinkage in 2002 , among which consumer theft accounts for 31.7 percent [4]. These thefts directly results in the lost of revenue and a smaller profit margin for retailers in an already highly competitive marketplace. And also, part of this burden is passed to customers via higher prices.

RFID can provide truly real-time and ubiquitous item tracking capability enhancing anti-theft applications. It enables a retailer to know which item has been moved and when. In addition, RFID can activate real-time alarms and trigger the locking of access doors. For example, by attaching a tag to the value item, such as a DV (Digital Video), the on-shelf reader reads the unique ID of the RFID tag and knows the existence of the protected item. In this case, passive RFID tags are more preferred considering the tradeoffs between the RFID solution cost and benefits. When the DV is moved out of the shelf, the reader will detect the missing of the enquired ID. The reader then transmits an alarm/warning signal to the back-end system, and initiates the other readers deployed in the warehouse to start looking for the missing tag. 
Whenever a reader locates the ID, a security monitor is triggered to video record the place where the item is newly detected. Almost every type of retail store can use this solution to protect its merchandise from being stolen by shoplifters.

\section{ii. Anti-counterfeiting}

Billions of dollars of revenue are lost due to counterfeiting. The most frequently counterfeited items are prescription drugs, currency bills and luxury items. For instance, the World Health Organization estimates that 5 to 8 percent of the drugs sold globally may be counterfeit, which would be a gray market up to nearly $\$ 30$ billion annually. RFID's can play a critical role to alleviate this problem. One RFID solution is to associate a unique item code with any particular prescription bottle so that it can be tracked through the supply chain. An ultra compact RFID tag proposed is shown in Fig. 6 for drug bottles tracking usage. The drug name, production site, expiration date, and even the description of the size/color of the container would be stored in the RFID IC chip. For example, after scanning, the following information would be displayed on the drug retailer's screen, "The drug is a yellow liquid inside a 3 inch high bottle. For example: Produced by Manufacturer X, plant Y in Athlone, Ireland. Expiration date Dec.1, 2007." If the information printed on the drug bottle has discrepancies in comparison to the one stored in the container, then we know counterfeiting happens. One 10-year forecast of RFID usage in the healthcare sector [5] is depicted in Fig. 7, showing the big potential of RFID in this area. For luxury items for example (e.g. fashion items); RFID can be used to prevent counterfeiting of brand name items. Unique ID can be embedded inside the items, such as fashion clothes. When such clothes surface outside an authorized selling region, the RFID tag can be used to track which reseller was responsible for distributing it to the gray market.

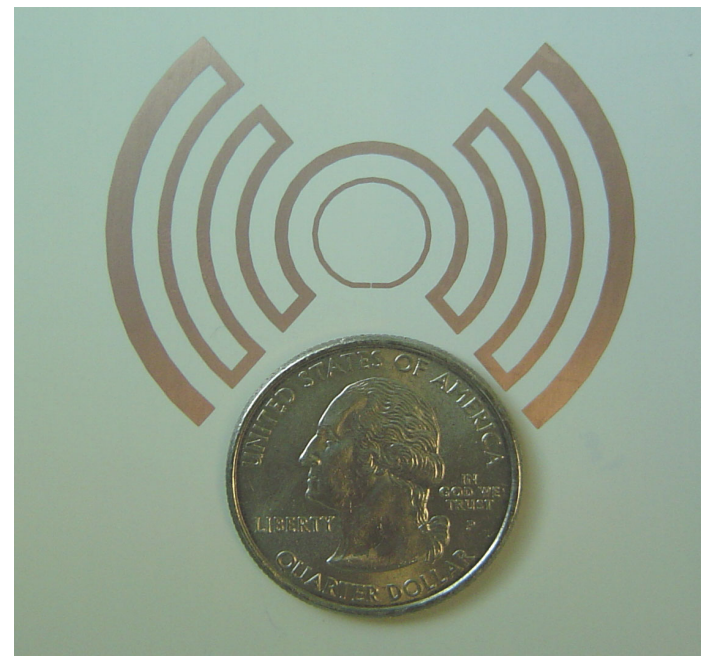

Fig. 6. An ultra compact RFID tag proposed for drug bottles

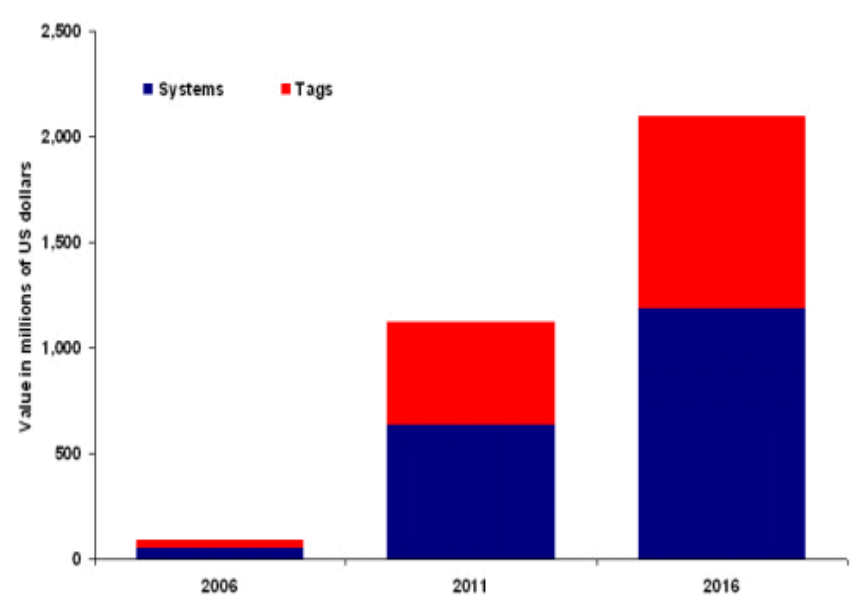

Fig. 7. 10-year forecast of RFID usage in the healthcare sector [5]

\section{iii. National security}

National security represents an extremely important concern. Hazardous materials can be hidden in cargos and shipped into major port cities by terrorists to launch attacks against a country. RFID can provide effective real-time monitoring to deter these types of attempts. Active RFID tags with rechargeable battery support have been proposed to label each container and track the item in global level utilizing GPS and "wireless security perimeter" configurations. When a container parks at an unauthorized area or experiences an unwanted intrusion/reload, the RFID tag will record such information and report to the information center, enhancing early alarm and warning efforts. 


\section{CONCLUSION}

In this paper, we proposed the development and implementation of novel paper-based UHF RFID designs for anticounterfeiting and security. Miniaturization and global-band antenna configurations and utilization of inkjet printing techniques in/on organic substrates, such as paper, that will enable the low cost and large-scale implementation of RFID-enabled semi-autonomous sensors for "cognitive-intelligence" applications, are also discussed for a variety of security-related areas, featuring a great potential for low-cost large-scale solutions.

\section{REFERENCES}

[1] "Design and Development of Novel Miniaturized UHF RFID Tags on Ultra-Low-Cost Paper-Based Substrates” L. Yang, S. Basat, A. Rida, and M. M. Tentzeris, APMC Dec 15, 2006.

[2] UHF Gen 2 System Overview, Online article, Available HTTP: http://tii.developerconference.ext.ti.com/postconf/downloads/rfid-tutorial2.pdf, viewed Feb 27, 2007

[3] D. Thompson, G. Ponchak, M. M. Tentzeris, J. Papapolymerou, "Characterization of LCP material and transmission lines on LCP substrates from 30 to 110GHz," IEEE Trans. Microwave Theory \& Tech., vol. 52, no. 4, pp. 1343-1352, April 2004.

[4] National Retail Security Survey, Final Report. University of Florida, 2002. Richard C. Hollinger, Ph.D., director, and Jason L. Davis, graduate research associate.

[5] Peter Harrop, and Raghu Das, "RFID in Healthcare 2006-2016”, IDTechEx, 2006. 